\title{
Reversibly controlled magnetic domains of Co film via electric field driven oxygen migration at nanoscale
}

Cite as: Appl. Phys. Lett. 114, 232401 (2019); https://doi.org/10.1063/1.5087964

Submitted: 06 January 2019. Accepted: 23 May 2019. Published Online: 10 June 2019

Pravarthana Dhanapal (D), Tuo Zhang, Baomin Wang (D), Huali Yang, Haicheng Xuan, Chong Bi, Weigang Wang, and Run-Wei Li

\section{ARTICLES YOU MAY BE INTERESTED IN}

Large spin-orbit torque observed in epitaxial $\mathrm{SrIrO}_{3}$ thin films

Applied Physics Letters 114, 232406 (2019); https://doi.org/10.1063/1.5097699

Magnetic skyrmions in atomic thin $\mathrm{Crl}_{3}$ monolayer

Applied Physics Letters 114, 232402 (2019); https://doi.org/10.1063/1.5096782

Vectorial observation of the spin Seebeck effect in epitaxial $\mathrm{NiFe}_{2} \mathrm{O}_{4}$ thin films with various magnetic anisotropy contributions

Applied Physics Letters 114, 232404 (2019); https://doi.org/10.1063/1.5092774

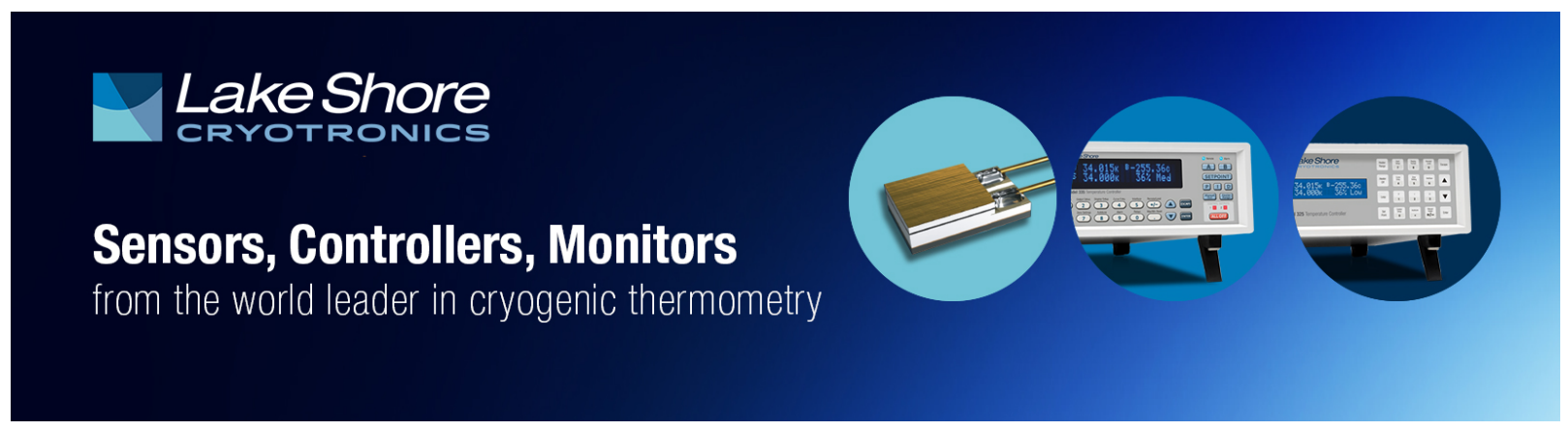




\title{
Reversibly controlled magnetic domains of Co film via electric field driven oxygen migration at nanoscale
}

Cite as: Appl. Phys. Lett. 114, 232401 (2019); doi: 10.1063/1.5087964

Submitted: 6 January 2019 - Accepted: 23 May 2019 .

Published Online: 10 June 2019
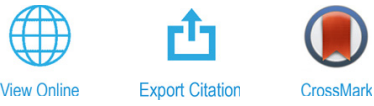

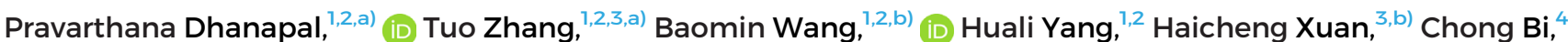
Weigang Wang, ${ }^{4}$ and Run-Wei $\mathrm{Li}^{1,2,5, \mathrm{~b})}$

\begin{abstract}
AFFILIATIONS
${ }^{7}$ CAS Key Laboratory of Magnetic Materials and Devices, Ningbo Institute of Materials Technology and Engineering, Chinese Academy of Sciences, Ningbo 315201, People's Republic of China

${ }^{2}$ Zhejiang Province Key Laboratory of Magnetic Materials and Application Technology, Ningbo Institute of Materials Technology and Engineering, Chinese Academy of Sciences, Ningbo 315201, People's Republic of China

${ }^{3}$ College of Materials Science and Engineering, Key Laboratory of Interface Science and Engineering in Advanced Materials, Ministry of Education, Taiyuan University of Technology, Taiyuan 030024, People's Republic of China

${ }^{4}$ Department of Physics, University of Arizona, Tucson, Arizona 85721, USA

${ }^{5}$ Center of Materials Science and Optoelectronics Engineering, University of Chinese Academy of Sciences, Beijing 100049, People's Republic of China
\end{abstract}

a) Contributions: P. Dhanapal and T. Zhang contributed equally to this work.

${ }^{b)}$ Authors to whom correspondence should be addressed: wangbaomin@nimte.ac.cn; xuanhaicheng@tyut.edu.cn; and runweili@nimte.ac.cn

\begin{abstract}
Electric field control of perpendicular magnetic anisotropy (PMA) can enable low power consumption for perpendicular magnetic random access memory devices. However, the tuning of PMA by the electric field in ferromagnetic metal thin films is less efficient and limited to the interface due to the screening effect. Alternatively, the magnetoionic effect can control PMA efficiently, which utilizes ion migrations over the surface of the ferromagnetic metal by interfacing it with the charge reservoir of oxygen ions like $\mathrm{GdO}_{\mathrm{x}}$. In this paper, we report the reversibly controlled magnetic domains of PMA Co in the $\mathrm{Pt} / \mathrm{Co} / \mathrm{GdO}_{\mathrm{x}}$ trilayer via the electric field at the nanoscale using conductive atomic force microscopy and magnetic force microscopy (MFM). The magnetic domain phase values determined by MFM decrease and increase when negative and positive bias voltages of magnitude $4 \mathrm{~V}$ are applied to the surface of $\mathrm{GdO}_{\mathrm{x}}$, respectively. These results suggest a path toward control of PMA materials at the nanoscale by the electric field for information storage devices.
\end{abstract}

Published under license by AIP Publishing. https://doi.org/10.1063/1.5087964

The tunable magnetic anisotropy of magnetic metal ultrathin films by static electric fields is of great interest to obtain low power magnetic random access memory (MRAM) devices for information storage applications. ${ }^{1-5}$ In particular, ferromagnetic metals with perpendicular magnetic anisotropy (PMA) are being investigated for perpendicular magnetic random access memory (p-MRAM) due to its better scalability and shape uniformity in comparison with in-plane MRAM. ${ }^{6}$ In this context, the stabilization of PMA in ferromagnetic metal ultrathin films and its control by the electric field are promising for efficient information storage devices. The PMA in ferromagnetic metals can be stabilized via interfacial spin-orbit coupling between heavy metal and ferromagnetic metal, leading to orbital ordering at the interface, for example, $\mathrm{Pt} / \mathrm{Co} / \mathrm{Pt}$ trilayer. ${ }^{7}$ The electric field control of such orbital ordering can lead to control of PMA such as electric driven charge accumulation and strain at the interface. ${ }^{8-10}$ However, the electric field controlled charge tuning is limited to only the interface due to the screening of free electrons in metals. ${ }^{11}$ Moreover, strain tuning of ferromagnetic metal relies on the interface with complex oxides. ${ }^{8}$ Alternatively, the $\mathrm{Pt} / \mathrm{metal} /$ oxide trilayer promising for thicker and thermally stable magnetic electrodes for p-MRAM with tunable magnetic anisotropy is possible by varying the oxidation time and annealing temperature during the deposition of the oxide layer over 
the Pt/Co films. ${ }^{12-16}$ Moreover, the amplitude of Pt/metal/oxide magnetic anisotropy is comparable to that of $\mathrm{Pt} / \mathrm{Co} / \mathrm{Pt}$ interfaces and exhibits lower magnetization pinning in comparison with $\mathrm{Pt} / \mathrm{Co} / \mathrm{Pt}$ interfaces, enabling it for high speed domain wall propagation for racetrack memories. ${ }^{1}$

Recently, Pt/Co interfaced with an amorphous gadolinium oxide $\left(\mathrm{GdO}_{\mathrm{x}}\right)$, which exhibits high oxygen mobility and acts as a reservoir of oxygen ions under the electric field, was explored for voltage control of PMA in all solid state devices. ${ }^{18-22}$ In the $\mathrm{Pt} / \mathrm{Co} / \mathrm{GdO}_{\mathrm{x}}$ system, the PMA can be switched reversibly in a nonvolatile fashion from out-ofplane to in-plane by voltage controlled reversible interfacial oxidation of the ultrathin Co film. The voltage-driven migration of oxygen in the $\mathrm{Pt} / \mathrm{Co} / \mathrm{GdO}_{\mathrm{x}}$ system is able to dramatically modify the magnetic properties of the Co layer, changing the magnetic anisotropy energy at the interface by $0.75 \mathrm{~mJ} \mathrm{~m}^{-2}$ at room temperature. Indeed, the electric field control coefficient $(\beta)$ of interfacial magnetocrystalline anisotropy is $>5000 \mathrm{fJ} /(\mathrm{V} \mathrm{m})$ in such systems, which is much higher than conventional magnetoelectric systems. ${ }^{17,23}$ In this work, we report the evolution of the magnetic domain in the PMA Co film with the electric field applied at the nanoscale by using scanning probe microscopy. The voltage modulation across $\mathrm{Co} / \mathrm{GdO}_{\mathrm{x}}$ is facilitated by the local application of the electric field by conductive atomic force microscopy (CAFM) over the ultrathin $\mathrm{Pt}(3 \mathrm{~nm})$ top electrode at room temperature. Subsequently, the effect of the electric field on stripe magnetic domains of PMA was investigated by magnetic force microscopy (MFM).

The $\mathrm{Pt} / \mathrm{Co} / \mathrm{GdO}_{\mathrm{x}}$ films were deposited on the thermally oxidized silicon substrate by $\mathrm{dc}$ magnetron sputtering at a base pressure of $5 \times 10^{-8}$ Torr. The continuous film structure is $\mathrm{Si} / \mathrm{SiO}_{2} / \mathrm{Pt}(4 \mathrm{~nm}) /$ $\mathrm{Co}(0.7 \mathrm{~nm}) / \mathrm{GdO}_{\mathrm{x}}(5 \mathrm{~nm})$ as shown in Fig. $1(\mathrm{a})$, where the number inside the parentheses is thickness in units of nanometer. A metal Gd layer of $\sim 1 \mathrm{~nm}$ thickness was first deposited before introducing oxygen into the sputtering chamber, which is a common method to avoid oxidation of under layers. ${ }^{24}$ After the oxidation of the Gd layer, the multilayers were vacuum annealed at $200^{\circ} \mathrm{C}$ for $10 \mathrm{~min}$ to enhance PMA. ${ }^{12}$ The hysteresis loops were measured by using a Superconducting Quantum Interference Device (SQUID). X-ray photoelectron spectra were obtained using an Axis Ultra delay-line-detector X-ray photoelectron spectrometer (XPS), using the $\mathrm{Mg} K_{\alpha}$ radiation source as the Xray source. An atomic force microscope was used to perform the MFM and C-AFM measurements. The thickness of $\mathrm{GdO}_{\mathrm{x}}$ is fixed to $5 \mathrm{~nm}$ to ensure minimal loss of MFM signals during the MFM measurements for the current study. Furthermore, the effect of toggling of oxygen migration at the interface $\mathrm{Co} / \mathrm{GdO}_{\mathrm{x}}$ on such a stable magnetic domain
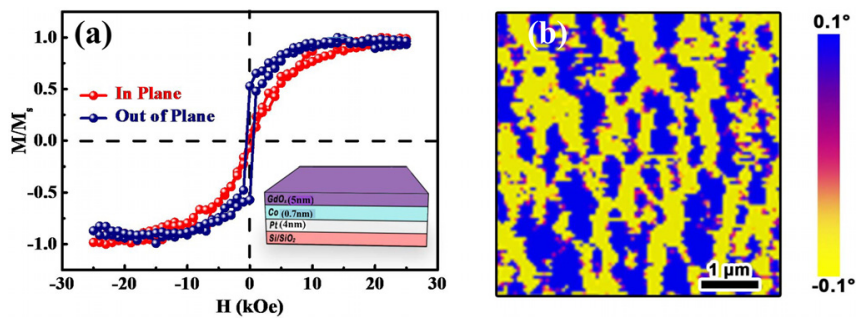

FIG. 1. (a) Normalized magnetic hysteresis loop along out-of-plane and in-plane sample directions at room temperature. The inset shows the schematic of the Pt/ $\mathrm{Co} / \mathrm{GdO}_{\mathrm{x}}$ trilayer sample system with the thickness of each layer indicated in parentheses. (b) MFM phase image. structure was driven by C-AFM, and subsequent magnetic changes were measured by MFM.

Figure 1(a) shows hysteresis loops of a continuous film measured by SQUID with magnetic fields applied along in-plane and perpendicular-to-plane orientation at room temperature. The magnetic hysteresis loop exhibits out-of-plane anisotropy with an anisotropy constant $\left(K_{\mathrm{u}}\right)$ of about $7.56 \times 10^{5} \mathrm{~J} \mathrm{~m}^{-3}$ and saturation magnetization $M_{\mathrm{s}}$ equal to $1.2 \times 10^{6} \mathrm{~A} \mathrm{~m}^{-1}$. The obtained $M_{\mathrm{s}}$ value is less than that of the pure Co film, which is equal to $1.43 \times 10^{6} \mathrm{~A} \mathrm{~m}^{-1}$. $^{25}$ The decrease in the $M_{\mathrm{s}}$ value could be attributed to partial oxidation of the Co film across the $\mathrm{Co} / \mathrm{GdO}_{\mathrm{x}}$ interface. The contribution of surface magnetic anisotropy $\left(K_{s}\right)$ was calculated to understand the origin of anisotropy. It was calculated from the following equation to understand the interfacial effect:

$$
K_{\mathrm{u}}=K_{\mathrm{v}}-\frac{\mu_{0} M_{\mathrm{s}}^{2}}{2}+\frac{K_{\mathrm{s}}}{t} .
$$

The calculated effective $K_{s}$ value of $0.6 \mathrm{~mJ} \mathrm{~m}^{-2}$ is obtained from Eq. (1), where $K_{v}$ is the bulk anisotropy constant equal to $8.2 \times 10^{5} \mathrm{~J} \mathrm{~m}^{-3}$ (Ref. 25) and $t$ is the thickness of the Co film. The effective $K_{s}$ value is the sum of surface anisotropy contributions from $\mathrm{Pt} / \mathrm{Co}$ and $\mathrm{Co} / \mathrm{GdO}_{\mathrm{x}}$ interfaces. The typical $K_{s}$ value for the Pt/Co interface varies in the range of $0.6-1.4 \mathrm{~mJ} \mathrm{~m}^{-2}$. 17 The value of $K_{s}$ is less than the previously obtained value of $1.3 \mathrm{~mJ} \mathrm{~m}^{-2}$ in a similar system. ${ }^{20}$ The reduction in effective $K_{s}$ could be attributed to the reduction in the interfacial contribution from $\mathrm{Co} / \mathrm{GdO}_{\mathrm{x}}$ because hybridization between sp-oxygen orbitals in $\mathrm{GdO}_{\mathrm{x}}$ and the $d_{z}^{2}$ Co orbital causes the origin of anisotropy across the $\mathrm{Co} / \mathrm{GdO}_{\mathrm{x}}$ interface. ${ }^{12,13,17}$ This reduction could be mainly attributed to the additional step of $\mathrm{Co} / \mathrm{GdO}_{\mathrm{x}}$ annealing performed in vacuum, which leads to the variation in the oxidation of the Co surface from $\mathrm{GdO}_{\mathrm{x}}$, and also evidenced by the decrease in the $M_{\mathrm{s}}$ value compared to the pure Co film. The typical stripe magnetic domain structure of PMA thin films has also been observed [Fig. (b).$^{26,27}$ This stripe domain structure occurs due to the minimizing demagnetization energy, which separates into up and down magnetization stripes.

The C-AFM and MFM investigations were carried out to understand the effect of the electric field at the nanoscale across the $\mathrm{Co} /$ $\mathrm{GdO}_{\mathrm{x}}$ interface. The C-AFM mobile probe can set bias independently over the surface of $\mathrm{GdO}_{\mathrm{x}}{ }^{18}$ It provides a more efficient oxygen ion transport process and has a great influence on magnetism. In order to make sure that the same region of the pristine magnetic domain structure recorded was subjected to electric bias, the C-AFM measurements were performed at room temperature to apply the local voltage bias to toggle the possible oxygen migration at the interface of $\mathrm{Co} / \mathrm{GdO}_{\mathrm{x}}$. After the C-AFM scan mode, we subsequently switch to the MFM mode to measure the magnetic domain structure. However, the MFM signal is sensitive to the surface conditions of the sample and susceptible to other disturbances such as van der Waals force and electrostatic force. ${ }^{28}$ The surface charge aggregation on the $\mathrm{GdO}_{\mathrm{x}}$ film after CAFM scan has a high influence on the magnetic domain signal. To reduce the impact of charge, we deposited the ultrathin Pt top layer of thickness $3 \mathrm{~nm}$ [Fig. 2(a)]. The thin Pt film has higher resistivity $\left(10^{-4}-10^{-1} \Omega \mathrm{m}\right)$ than bulk $\mathrm{Pt}\left(10^{-7} \Omega \mathrm{m}\right)$, such that upon application of bias, the movement of charge carriers is limited, and the area of application is only limited to the area of the C-AFM tip radius of $30 \mathrm{~nm}^{29}$ So the set bias on the thin Pt film is also localized in a short 
(a)
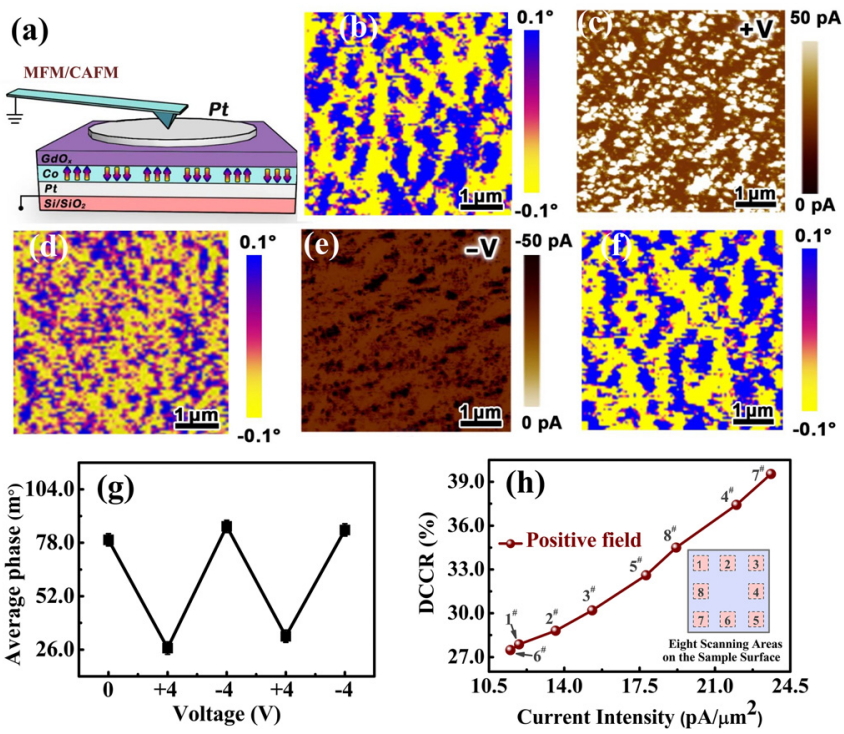

FIG. 2. (a) The schematic of the experimental setup to perform MFM/C-AFM over the top ultrathin $\mathrm{Pt}$ layer over $\mathrm{Pt} / \mathrm{Co} / \mathrm{GdO}_{\mathrm{x}}$. (b) MFM domain phase image in the pristine state. (c) C-AFM tunable current image for a bias voltage of $+4 \mathrm{~V}$. (d) MFM domain phase image after $+4 \mathrm{~V}$ bias voltage. (e) C-AFM tunable current image for a bias voltage of $-4 \mathrm{~V}$. (f) MFM domain phase image after $-4 \mathrm{~V}$ bias voltage. (g) The plot of the average magnetic domain phase value starting from the pristine state to alternative positive and negative bias voltage of magnitude $4 \mathrm{~V}$. (h) The plot of DCCR vs current intensities for positive voltage.

time during the C-AFM scan. The thin Pt top layer does not have an influence on the magnetic contrast of the Co PMA layer because the domain structure can be observed as shown in Fig. 2(b). Figure 2(c) shows the region of current flow in bright white regions, and the bright regions reveal upward current flow. The current flow can be mainly attributed to the downward movement of negative oxygen anions downward and positive holes upward. Subsequently, the magnetic domain structure observed by MFM as shown in Fig. 2(d) exhibits a decrease in the domain contrast over the region of current flow (oxygen migration). In MFM, the magnetic probe detects only the sample magnetic dipole with the magnetization direction perpendicular to the sample surface, and any decrease in phase contrast could be attributed to the decrease in the local anisotropy of magnetic domains. This means that local anisotropy of the magnetic domains under positive bias decreases due to the decrease in the measured phase value (stray magnetic field) along out-of-plane, which is proportional to the magnetic moment (saturation magnetization) of the sample. ${ }^{30,31}$ Further application of negative bias can move back $\mathrm{O}^{2-}$ from the Co film to the interface of the $\mathrm{Co} / \mathrm{GdO}_{\mathrm{x}}$ surface. Indeed, the dark region that represents downward current flow with oxygen anions moves upward as shown in Fig. 2(e). As a result, the oxygen anion moving upward leads to revival of magnetic domain contrast in the out-of-plane direction [Fig. 2(f)].

We also analyzed the magnetic and conductive domain structures starting from the pristine state to subsequent bias voltages to understand qualitatively the effect of oxygen migration on the nanoscale PMA modulation. The phase value decreases after positive bias and increases after negative bias in two cycles as shown in Fig. $2(\mathrm{~g})$.
Moreover, the phase value after negative bias is little higher than the pristine state. This could be attributed to the partial amount of Co film oxidization in the pristine state during the deposition of $\mathrm{GdO}_{\mathrm{x}}$. Furthermore, we performed the analysis for randomly eight regions of different current densities with the corresponding domain contrast change ratio $[$ DCCR $=$ (initial domain phase value - domain phase value after bias)/initial domain phase value] as shown in Fig. 2(h). It follows a linear relationship with the increase in the current density, leading to the increase in the DCCR. This implies that more oxygen migrations take place with the increase in the current density, which leads to enhanced DCCR. A similar trend was followed for negative bias, indicating that electric driven oxygen migrations controlled magnetic domain changes.

Figures 3(a) and 3(b) show that the effect of C-AFM scan cycles influences current flow and its effect on magnetic domain changes. The continuous C-AFM scan cycles of set positive bias voltage $2 \mathrm{~V}$ result in more current intensity. This could be attributed to more migration of oxygen ions due to vacancies as confirmed in the later section by XPS, which results in the reduction of local magnetic anisotropy as indicated by the decrease in the phase contrast with scan cycles in Fig. 3(c) ${ }^{18}$ But the average phase value increased in the second cycle, which reveals that there are more oxygen ions driven into the Co surface, which increases the PMA locally. ${ }^{21}$ Indeed, the DCCR increases with scan cycles, which indicates that more oxygen moves into the Co film as shown in Fig. 3(d). The time at each pixel in the CAFM scan is $\sim 6 \mathrm{~ms}$, which is calculated by using the scan rate of $0.5 \mathrm{~Hz}$ (for one sample scan line, it takes $1 \mathrm{~s}$ ). The time scale of magnetic changes seen at the nanoscale is shorter in comparison with the prolonged voltage application time on the order of minutes to induce
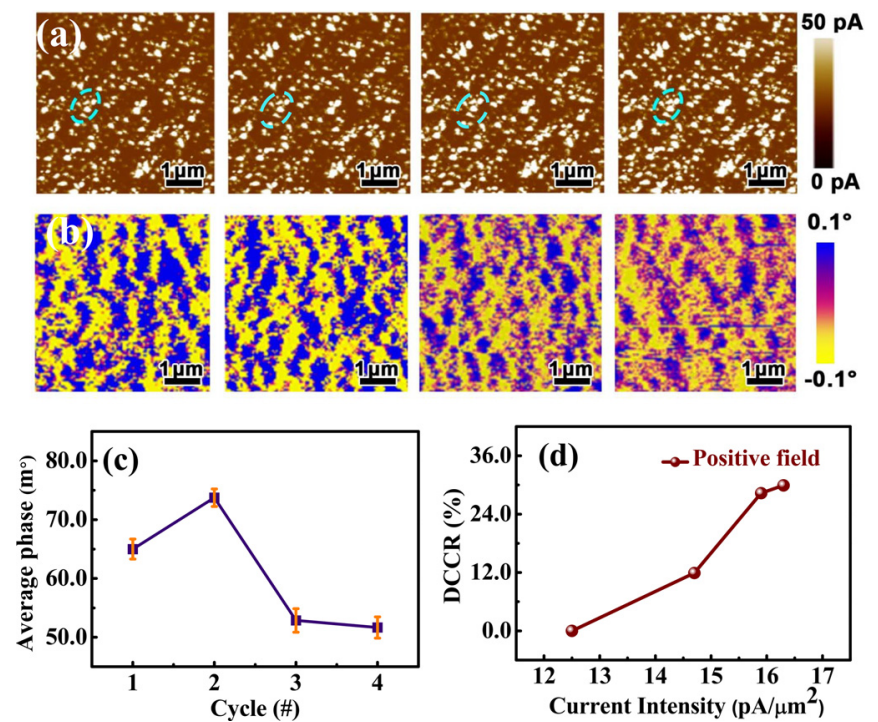

FIG. 3. (a) Images of C-AFM tunable current scan under a bias voltage of $+2 \mathrm{~V}$ with repeatable scan, starting first scan from left to right with the increase in the scan cycles. The dotted aqua blue circles indicate the same region from the first to fourth scan. (b) The corresponding MFM domain phase region shown below for (a) with the increase in scan cycles from left to right. (c) The plot of the average magnetic domain phase value vs scan cycles. (d) The plot of DCCR vs Current intensity. 

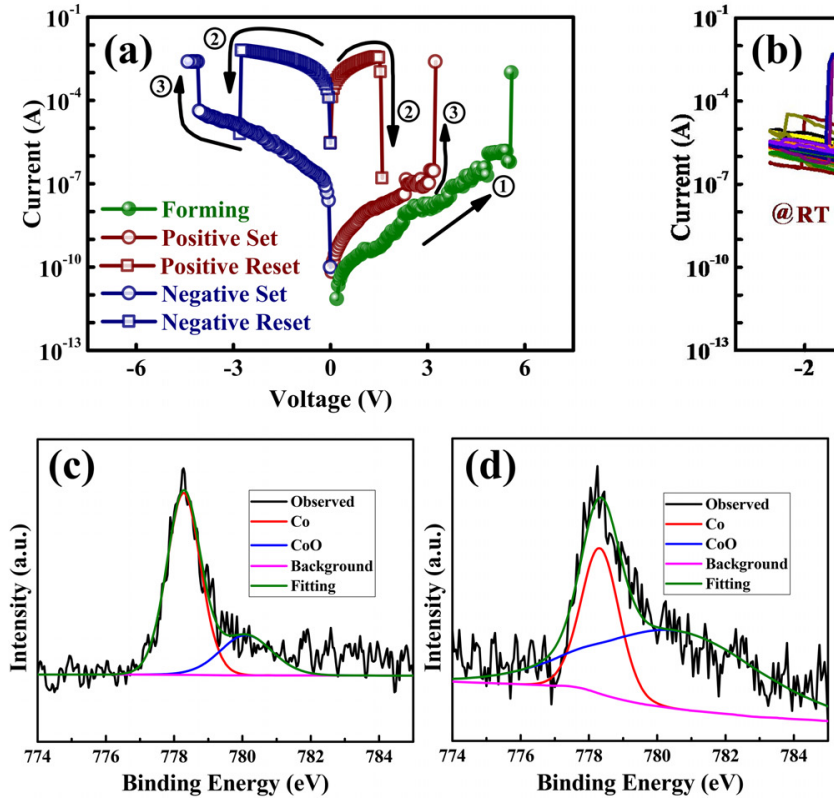

the magnetization reversal probably due to stabilization of large oxygen vacancies in $\mathrm{GdO}_{\mathrm{x}}$ due to vacuum annealing. ${ }^{24}$ The value of power involved for modulation of the stray field of the magnetic domain is $0.25 \mathrm{pW}$. The power is calculated from $I^{2} R$, where $\mathrm{I}$ is the average current value read from the C-AFM measurement $(\sim 50 \mathrm{pA})$ and $R$ is the sample resistance at $2 \mathrm{~V}\left(\sim 10^{8} \Omega, R=d V / d I\right)$.

To further study the electric field induced oxygen migration behavior of $\mathrm{GdO}_{\mathrm{x}}, \mathrm{Pt} / \mathrm{Co} / \mathrm{GdO}_{\mathrm{x}}$ with a top thick Pt electrode diameter of $100 \mu \mathrm{m}$ was fabricated [inset of Fig. 4(b)]. Figure 4(a) shows the resistive switching behavior of $\mathrm{GdO}_{\mathrm{x}}$ by sweeping the applied voltage. The forming voltage ( $V_{\text {forming }}$ ) of the $\mathrm{GdO}_{\mathrm{x}}$ film is about $5.8 \mathrm{~V}$ as indicated with operation (1), which initiates the low resistive state (LRS) in the $\mathrm{GdO}_{\mathrm{x}}$ insulator. Furthermore, the reset voltage $\left(V_{\text {reset }}\right)$ of $1.8 \mathrm{~V}$ and set voltage $\left(V_{\text {set }}\right)$ of $3 \mathrm{~V}$ are determined for LRS to the high resistive

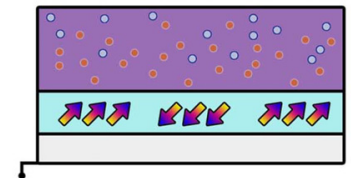

Initial State

$\square: \mathrm{GdO}_{\mathrm{x}} \quad \square: \mathrm{Co} \quad \square: \mathrm{Pt}$

$O:$ Oxygen Vacancy $\bullet:$ : Oxygen lon

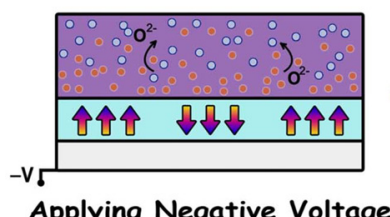

Applying Negative Voltage

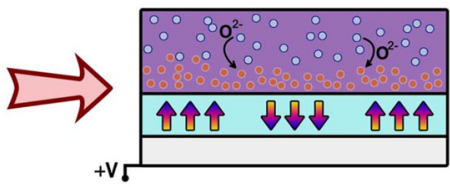

Applying Positive Voltage
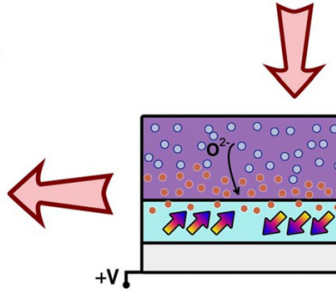

Applying Continuously Positive Voltage

FIG. 5. The schematic of the mechanism of nanoscale modulation of PMA by electric driven reversible toggling of oxygen migrations at the interface of $\mathrm{Co} / \mathrm{GdO}_{\mathrm{x}}$.

state (HRS) and HRS to LRS, respectively. The operation sequence is indicated with arrows 2 and 3 in Fig. 4(a) with a sudden decrease (operation (2) and increase (operation (3) in the current values occurred at $1.8 \mathrm{~V}$ and $3 \mathrm{~V}$, respectively. Similarly, under negative bias, the switching state changes to HRS from LRS in operation (2) at $-2.8 \mathrm{~V}$ and reverses back to LRS from HRS in operation (3) at $-4 \mathrm{~V}$. A similar trend of switching back of HRS to LRS or vice versa is observed from both positive and negative sides of bias voltages for many cycles as shown in Fig. 4(b). With an increase in voltage from $0 \mathrm{~V}$, it can be seen that a set process occurs at set voltage, which triggers an abrupt change from HRS to LRS and vice versa for reset voltage. The resistances of the two states scatter in a certain range during 20 cycles, ensuring that the switching between HRS and LRS is highly repeatable and reliable. ${ }^{32}$ The time scale for full reversibility of resistive switching states occurs on the order of few milliseconds. This result is similar to the previous reports on the resistive switching characteristics exhibited in the $\mathrm{Ti} / \mathrm{Gd}_{2} \mathrm{O}_{3} / \mathrm{Pt}$ structure, and such resistive switching phenomena are attributed to migration of oxygen ions. ${ }^{33}$ To examine the effect of voltage bias on the oxidation of the Co film, we performed XPS as it can detail the oxidation state of the Co film qualitatively and quantitatively. ${ }^{16,17,34}$ The XPS Co $2 \mathrm{p}_{3 / 2}$ core level spectra of Co films were recorded in different states of Co films such as the pristine state, after 10 resistive switching cycles stopped in LRS and HRS as shown in Figs. $4(\mathrm{c})-4(\mathrm{e})$. In all the states, Co $2 \mathrm{p}_{3 / 2}$ core level spectra can be fitted into two components; one is the metallic Co peak at $278 \mathrm{eV}$, and the other is the oxidized $(\mathrm{CoO})$ peak at $280 \mathrm{eV}$ as indicated by red and blue curves, respectively. ${ }^{34}$ In the pristine state, the ratio of $\mathrm{CoO} / \mathrm{Co}$ concentration is 0.33 , which indicates that the as-deposited state is partially oxidized. After LRS, the $\mathrm{CoO} / \mathrm{Co}$ ratio increases to 2.2, which indicates that under positive voltage bias, more oxygen ions migrate toward the Co film, resulting in the increase in the $\mathrm{CoO}$ concentration, while after HRS, the $\mathrm{CoO} / \mathrm{Co}$ ratio decreases to 1.6 due to the decrease in the $\mathrm{CoO}$ concentration. Thus, the resistive switching under positive/negative voltage bias could be attributed to oxygen ion migrations. 
Figure 5 depicts a schematic of the mechanism of nanoscale modulation of magnetic domains measured by MFM under the electric field driven by C-AFM for reversible toggling of oxygen migrations at the interface of $\mathrm{Co} / \mathrm{GdO}_{\mathrm{x}}$. When positive (negative) sample bias is set, negatively charged oxygen ions $\left(\mathrm{O}^{2-}\right)$ are attracted (repelled) toward the positive pole, and $\mathrm{O}^{2-}$ moves close (far away) to the $\mathrm{Co} /$ $\mathrm{GdO}_{\mathrm{x}}$ surface. It can be seen that the positive bias decreases the magnetic domain phase value, while the opposite is observed for negative bias; when bias is applied for a prolonged time, oxygen could migrate beyond the interface. ${ }^{23}$ This indicates that the PMA of the Co film is reduced under positive bias, while the PMA state returns to the original state under negative bias.

In summary, in situ electric field control of magnetic domain changes of $\mathrm{Pt} / \mathrm{Co} / \mathrm{GdO}_{\mathrm{x}}$ is achieved at room temperature. The magnetic domain phase values determined by MFM decrease (increase) in the perpendicular to plane magnetic anisotropy when oxygen is driven into (out of) the Co film via electrically induced migration of oxygen ions from $\mathrm{GdO}_{\mathrm{x}}$ by C-AFM locally. These results suggest a path toward control of PMA materials at the nanoscale based on the magnetoionic effect.

This work was supported by the National Key R\&D Program of China (No. 2016YFA0201102), the National Natural Science Foundation of China (Nos. 51871232, 51571208, 51525103, and 6171101158), the Youth Innovation Promotion Association of the Chinese Academy of (No. 2016270), the Key Research Program of the Chinese Academy of Sciences (No. KJZD-EW-M05), the Ningbo Science and Technology Bureau (2025 S\&T Megaprojects), and the Ningbo Science and Technology Innovation Team (No. 2015B11001).

\section{REFERENCES}

${ }^{1}$ W. G. Wang, M. Li, S. Hageman, and C. L. Chien, Nat. Mater. 11, 64 (2012).

${ }^{2}$ M. Weisheit, S. Fähler, A. Marty, Y. Souche, C. Poinsignon, and D. Givord, Science 315, 349 (2007).

${ }^{3}$ N. N. Negulyaev, V. S. Stepanyuk, W. Hergert, and J. Kirschner, Phys. Rev. Lett. 106, 037202 (2011).

${ }^{4}$ C. G. Duan, J. P. Velev, R. F. Sabirianov, Z. Zhu, J. Chu, S. S. Jaswal, and E. Y. Tsymbal, Phys. Rev. Lett. 101, 137201 (2008).

${ }^{5}$ K. Nakamura, R. Shimabukuro, Y. Fujiwara, T. Akiyama, T. Ito, and A. J. Freeman, Phys. Rev. Lett. 102, 187201 (2009).

${ }^{6}$ S. Bhatti, R. Sbiaa, A. Hirohata, H. Ohno, S. Fukami, and S. N. Piramanayagam, Mater. Today 20, 530 (2017).

${ }^{7}$ F. Hellman, A. Hoffmann, Y. Tserkovnyak, G. S. D. Beach, E. E. Fullerton, C. Leighton, A. H. MacDonald, D. C. Ralph, D. A. Arena, H. A. Dürr, P. Fischer, J. Grollier, J. P. Heremans, T. Jungwirth, A. V. Kimel, B. Koopmans, I. N. Krivorotov, S. J. May, A. K. Petford-Long, J. M. Rondinelli, N. Samarth, I. K.
Schuller, A. N. Slavin, M. D. Stiles, O. Tchernyshyov, A. Thiaville, and B. L. Zink, Rev. Mod. Phys. 89, 025006 (2017).

${ }^{8}$ G. Radaelli, D. Petti, E. Plekhanov, I. Fina, P. Torelli, B. R. Salles, M. Cantoni, C. Rinaldi, D. Gutiérrez, G. Panaccione, M. Varela, S. Picozzi, J. Fontcuberta, and R. Bertacco, Nat. Commun. 5, 1 (2014).

${ }^{9}$ Y. Sun, Y. Ba, A. Chen, W. He, W. Wang, X. Zheng, L. Zou, Y. Zhang, Q. Yang, L. Yan, C. Feng, Q. Zhang, J. Cai, W. Wu, M. Liu, L. Gu, Z. Cheng, C.-W. Nan, Z. Qiu, Y. Wu, J. Li, and Y. Zhao, ACS Appl. Mater. Interfaces 9, 10855 (2017).

${ }^{10}$ C. Song, B. Cui, F. Li, X. Zhou, and F. Pan, Prog. Mater. Sci. 87, 33 (2017).

${ }^{11}$ F. Matsukura, Y. Tokura, and H. Ohno, Nat. Nanotechnol. 10, 209 (2015).

${ }^{12}$ L. E. Nistor, B. Rodmacq, S. Auffret, and B. Dieny, Appl. Phys. Lett. 94, 012512 (2009).

${ }^{13}$ Q.-L. Lv, J.-W. Cai, H.-Y. Pan, and B.-S. Han, Appl. Phys. Express 3, 093003 (2010).

${ }^{14}$ B. Rodmacq, A. Manchon, C. Ducruet, S. Auffret, and B. Dieny, Phys. Rev. B 79, 024423 (2009).

${ }^{15}$ S. Monso, B. Rodmacq, S. Auffret, G. Casali, F. Fettar, B. Gilles, B. Dieny, and P. Boyer, Appl. Phys. Lett. 80, 4157 (2002).

${ }^{16}$ A. Manchon, C. Ducruet, L. Lombard, S. Auffret, B. Rodmacq, B. Dieny, S. Pizzini, J. Vogel, V. Uhlír, M. Hochstrasser, and G. Panaccione, J. Appl. Phys. 104, 043914 (2008).

${ }^{17}$ B. Dieny and M. Chshiev, Rev. Mod. Phys. 89, 025008 (2017).

${ }^{18}$ U. Bauer, S. Emori, and G. S. D. Beach, Nat. Nanotechnol. 8, 411 (2013).

${ }^{19}$ U. Bauer, L. Yao, A. J. Tan, P. Agrawal, S. Emori, H. L. Tuller, S. van Dijken, and G. S. D. Beach, Nat. Mater. 14, 174 (2015).

${ }^{20}$ U. Bauer, S. Emori, and G. S. D. Beach, Appl. Phys. Lett. 101, 172403 (2012).

${ }^{21}$ C. Bi, C. Sun, M. Xu, T. Newhouse-Illige, P. M. Voyles, and W. Wang, Phys. Rev. Appl. 8, 034003 (2017).

${ }^{22}$ X. Zhou, Y. Yan, M. Jiang, B. Cui, F. Pan, and C. Song, J. Phys. Chem. C 120, 1633 (2016).

${ }^{23}$ D. A. Gilbert, A. J. Grutter, E. Arenholz, K. Liu, B. J. Kirby, J. A. Borchers, and B. B. Maranville, Nat. Commun. 7, 12264 (2016).

${ }^{24}$ C. Bi, Y. Liu, T. Newhouse-Illige, M. Xu, M. Rosales, J. W. Freeland, O. Mryasov, S. Zhang, S. G. E. Te Velthuis, and W. G. Wang, Phys. Rev. Lett. 113, 267202 (2014).

${ }^{25}$ F. Virot, L. Favre, R. Hayn, and M. D. Kuz'min, J. Phys. D: Appl. Phys. 45, 405003 (2012).

${ }^{26}$ B. Kaplan, J. Magn. Magn. Mater. 298, 135 (2006).

${ }^{27}$ M. Kronseder, M. Buchner, H. G. Bauer, and C. H. Back, Nat. Commun. 4, 2054 (2013).

${ }^{28}$ C. Liu, J. Ma, J. Ma, Y. Zhang, J. Chen, and C. W. Nan, AIP Adv. 7, 055003 (2017).

${ }^{29}$ L. J. McGilly, P. Yudin, L. Feigl, A. K. Tagantsev, and N. Setter, Nat. Nanotechnol. 10, 145 (2015).

${ }^{30}$ F. Bonell, Y. T. Takahashi, D. D. Lam, S. Yoshida, Y. Shiota, S. Miwa, T. Nakamura, and Y. Suzuki, Appl. Phys. Lett. 102, 152401 (2013).

${ }^{31}$ P. Dhanapal, S. Guo, B. Wang, H. Yang, S. Agarwal, Q. Zhan, and R.-W. Li, Appl. Phys. Lett. 111, 162401 (2017).

${ }^{32}$ G. Chen, C. Song, C. Chen, S. Gao, F. Zeng, and F. Pan, Adv. Mater. 24, 3515 (2012).

${ }^{33}$ K.-C. Liu, W.-H. Tzeng, K.-M. Chang, Y.-C. Chan, C.-C. Kuo, and C.-W. Cheng, Microelectron. Reliab. 50, 670 (2010).

${ }^{34}$ X. Chen, C. Feng, Z. L. Wu, F. Yang, Y. Liu, S. Jiang, M. H. Li, and G. H. Yu, Appl. Phys. Lett. 104, 052413 (2014). 